Check for updates

Cite this: Chem. Sci., 2019, 10, 5929

๑ All publication charges for this article have been paid for by the Royal Society of Chemistry

Received 24th January 2019

Accepted 3rd May 2019

DOI: $10.1039 / \mathrm{c} 9 \mathrm{sc} 00403 \mathrm{c}$

rsc.li/chemical-science

\section{Chemically modified nanofoci unifying plasmonics and catalysis $\uparrow$}

\author{
Yueliang Wang, ${ }^{a}$ Lingling Fang, ${ }^{a}$ Ming Gong ${ }^{b}$ and Zhaoxiang Deng (D) *a
}

A plasmonic nanofocus, often in the form of a nanogap, is capable of concentrating light in a nanometric volume. The greatly enhanced electromagnetic field offers many opportunities in physics and chemistry. However, the lack of a method to fine-tune the chemical activities of the nanofocus has severely limited its application. Here we communicate an intriguing class of chemically modified nanofoci (CMNFs) that are able to address this challenge. Our results successfully demonstrate a possibility to functionalize the nanosized, mass-transport-restricted nanogap (nanofocus) of a dimeric gold nanoparticle assembly with homo-( $\mathrm{Au}$ ) and heterogeneous ( $\mathrm{Ag}, \mathrm{Pt}$, and $\mathrm{Pd}$ ) materials. The as-produced structures with conductive $\mathrm{Au}$ and $\mathrm{Ag}$ junctions generate a novel form of charge transfer plasmon (CTP) with continuously tunable frequency covering the visible and near-infrared domains. In addition, the Ag materials can be displaced by catalytic Pt and Pd metals while still maintaining a tightly focused electromagnetic field. These hybrid structures with unified catalytic and plasmonic properties enable real-time, on-site probing of catalytic conversions at the nanofocus by plasmon-enhanced Raman scattering. The chemically/optically engineered CMNFs represent the simplest function-integrated nanodevices for plasmonics, sensing, and catalysis. Our work not only realizes chemical CTP reshaping, but also allows chemical functionalization into an intensified plasmonic near-field. The latter may enable unconventional chemical reactions driven by the catalytically functionalized, strongly boosted light field.

\section{Introduction}

Light-excited collective and coherent movements of conduction electrons in a metal structure, termed localized surface plasmon resonance (LSPR), ${ }^{1-3}$ offer great opportunities in ultrafast optical nanocircuitry, ${ }^{4,5}$ information transport, ${ }^{6,7}$ light harvesting, ${ }^{8,9}$ sensing, ${ }^{10-12}$ nanomedicine, ${ }^{13,14}$ and various hot-electronmediated processes. ${ }^{15-17}$ Particularly, the LSPRs of nanoparticles (NPs) in close vicinity can hybridize into new resonance modes. ${ }^{18-20}$ In the case of gold nanoparticle (AuNP) dimers with a small dielectric gap, a bonding dipolar plasmon (BDP) exists at a low energy level due to strongly coupled electric dipoles. ${ }^{18-21}$ The BDP results in charge piling-up and sub-diffractional light trapping (nanofocus) inside the nanogap..$^{22-25}$ Once a capacitive nanogap is shorted, charge neutralization will happen, accompanied by the formation of a charge transfer plasmon (CTP). ${ }^{20,21,24-27}$ The CTP delivers a facile way for plasmonic tuning well into the near infrared (NIR) domain, ${ }^{24-27}$ signaling

\footnotetext{
${ }^{a}$ CAS Key Laboratory of Soft Matter Chemistry, Hefei National Research Center for Physical Sciences at the Microscale, Department of Chemistry, University of Science and Technology of China, Hefei, Anhui 230026, China.E-mail: zhxdeng@ustc.edu.cn ${ }^{b}$ Engineering and Materials Science Experiment Center, University of Science and Technology of China, Hefei, Anhui 230027, China

$\dagger$ Electronic supplementary information (ESI) available: Methods and extra supporting figures. See DOI: 10.1039/c9sc00403c
}

the electron transport properties of the junction materials at optical frequencies. ${ }^{26,28}$

Compared to photolithography, solution-processing techniques are superior in the composition, crystallinity, and 3D shape control of plasmonic nanostructures. ${ }^{29-34}$ In particular, DNA nanotechnology has enabled programmable assembly of inorganic building blocks into prescribed structures..$^{35-37}$ However, a strong capacitive coupling or direct charge transfer is often prohibited in these assemblies by the bulky DNA strands. ${ }^{38,39}$ To circumvent this problem, dried samples (unexpected due to structural disruption) are often used, as capillary forces generated during water evaporation can bring NPs in close contact. ${ }^{40-42}$ Apart from self-assembly, direct synthesis of interfaced plasmonic nanodimers is possible but producing gapped structures is hard. ${ }^{29-31} \mathrm{~A}$ method based on combined optical-electrochemical processes has been reported for in situ growth of NP patterns. ${ }^{43,44}$ However, it is difficult to achieve spacing control with nanometer accuracy. We developed a facile and highly efficient $\mathrm{Ag}$ ion soldering (AIS) strategy to make subnm-gapped nanofoci. ${ }^{45}$ The strong coupling enabled by AIS and its adaptability to DNA nanotechnology provided a tool to realize hybridized LSPRs of plasmonic "molecules". ${ }^{46}$ Upon thermal reshaping, tunable CTPs up to the NIR II regime were obtained. ${ }^{47,48}$ However, the lack of chemical tunability severely limited the application of the nanofoci. To surpass this barrier, spatially addressable chemical modifiability should be achieved 
for the subnm gaps without compromising on their light focusing ability.

Herein we report a novel class of chemically modified nanofoci (CMNFs) as the smallest compound (dimer) structures with unified plasmonic/catalytic functions (Scheme 1). From a kinetic point of view, the hindered mass transport to the ultranarrow and recessed nanogap of the AuNP dimer does not favor chemical modification (e.g. metal deposition) inside the nanofocus. Favorably, thermodynamics supports gap filling to minimize the dimer's interfacial energy. Accordingly, the CMNFs can be formed under properly chosen conditions to overcome the kinetic barrier. In this process, the subnm gap achieved by AIS is highly beneficial to prime the junction growth. The tunable chemical activities of the CMNFs clearly distinguish them from structures made by purely physical processes. ${ }^{4,48}$ The resulting Au or Ag conductive junction (CJ) in a dimer allows highly efficient CTP reshaping. The Ag CJ can be converted into $\mathrm{Pt} / \mathrm{Pd}$ catalysts via galvanic displacement, enabling surface enhanced Raman spectroscopy (SERS) monitoring of catalytic reactions at the nanofocus. ${ }^{49,50}$ Therefore, the catalytic activities provided by the in-hotspot metal deposits and the plasmon-enabled SERS probing can work together cooperatively. In particular, the CMNFs with definable plasmonic and catalytic properties may help in the pursuit of new chemistry in the strongly boosted optical near-field. ${ }^{51-53}$ Note that direct assembly of catalytic Pt or Pd-based NPs as in our previous work could not generate intensified light fields for the above applications due to their heavily damped plasmon resonances. ${ }^{45}$

\section{Results and discussion}

Bis( $p$-sulfonatophenyl) phenyl phosphine (BSPP) capped AuNPs with diameters of 23,30 , and $43 \mathrm{~nm}$ (ESI, Fig. S1 $\dagger$ ) were employed to make nanodimers via an AIS process. ${ }^{45}$ The resulting AuNP clusters were electrophoresed in an agarose gel to isolate dimeric assemblies (ESI, Fig. S2 $\dagger$ ). The AIS relies on ligand stripping from the AuNPs driven by the formation of a stable $\mathrm{BSPP}-\mathrm{Ag}^{+}$complex. ${ }^{45}$ The resulting ligand-deficient AuNPs are very "sticky", which immediately form discrete clusters in the presence of a fish sperm DNA stabilizer. Extinction spectra (ESI, Fig. S3†) suggested strong coupling of the gelpurified AuNP dimers (ESI, Fig. S4 $\dagger$ ) according to their BDP wavelengths. Computations revealed an interparticle gap of

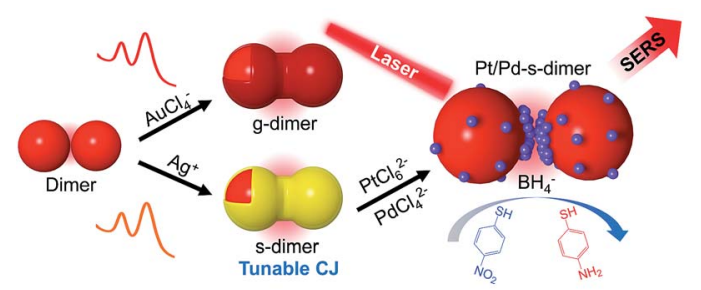

Scheme 1 Schematic illustration of a chemical route to realize gapengineered AuNP dimers for charge transfer plasmon reshaping and in-hotspot catalytic reaction.
0.65-0.9 nm (ESI, Fig. S3(d)†). Therefore, the bonding between NPs could be attributed to van der Waals attractive forces within a sub-nm distance based on the DLVO theory. ${ }^{54}$

The super-narrow interparticle separation would facilitate nucleation of the $\mathrm{Au}$ or $\mathrm{Ag}$ junction in the dimeric gap during metal deposition. However, failures were experienced during our initial attempts (ESI, Fig. S5-S7†). We reasoned that a high reaction temperature would be able to increase the diffusivity of surface-deposited metal atoms to enter the gap areas, and enhance diffusional and convectional transports of chemicals into the gap vicinities. At an elevated temperature, desorption of FSDNA from the AuNPs would happen, followed by citrate backfilling to provide an activated surface for the metal deposition. Accordingly, Au and Ag conductive junctions (CJs) could be formed in the nanogaps with citrate ions as both the reducing and surface-capping agent. Due to the gap-preferred metal deposition, the CJs were controllable in width by simply altering $\mathrm{HAuCl}_{4}$ and $\mathrm{AgNO}_{3}$ concentrations (Fig. 1 and 2, panels a-c). The resulting CMNFs with gold and silver CJs were termed g-dimers and s-dimers, respectively. We measured the widths of the CJs and the diameters of the metal-deposited AuNPs based on transmission electron microscopy (TEM) images. The statistical data in ESI Fig. S8-S11† suggested more Au and Ag deposits in the nanofoci, possibly due to surface atom rearrangements. The gap-directed metal deposition was more
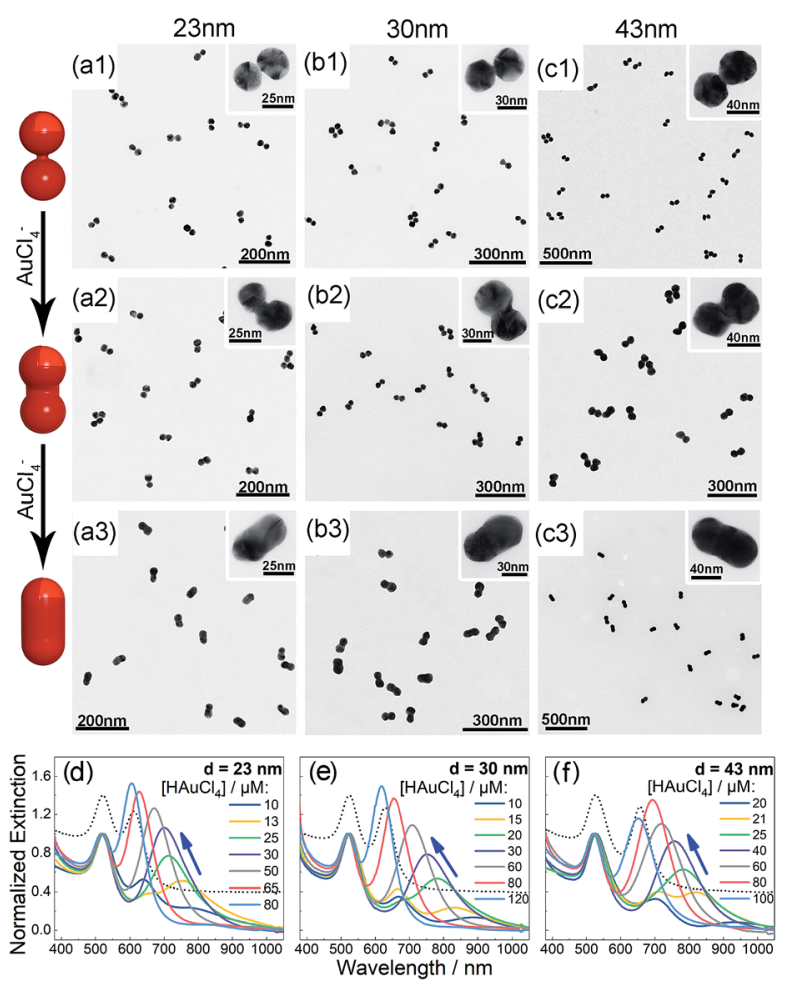

Fig. 1 (a-c) TEM images of the as-synthesized g-dimers with increased gold deposition (1-3) based on 23 (a), 30 (b), and $43 \mathrm{~nm}$ (c) AuNPs. Insets are magnified views of the CMNFs. (d-f) Extinction spectra of AuNP dimers after gold deposition to form tunable CTPs. Dotted curves indicate the BDP peaks (located at the right side of the $520 \mathrm{~nm}$ resonances) of unmodified dimers. 


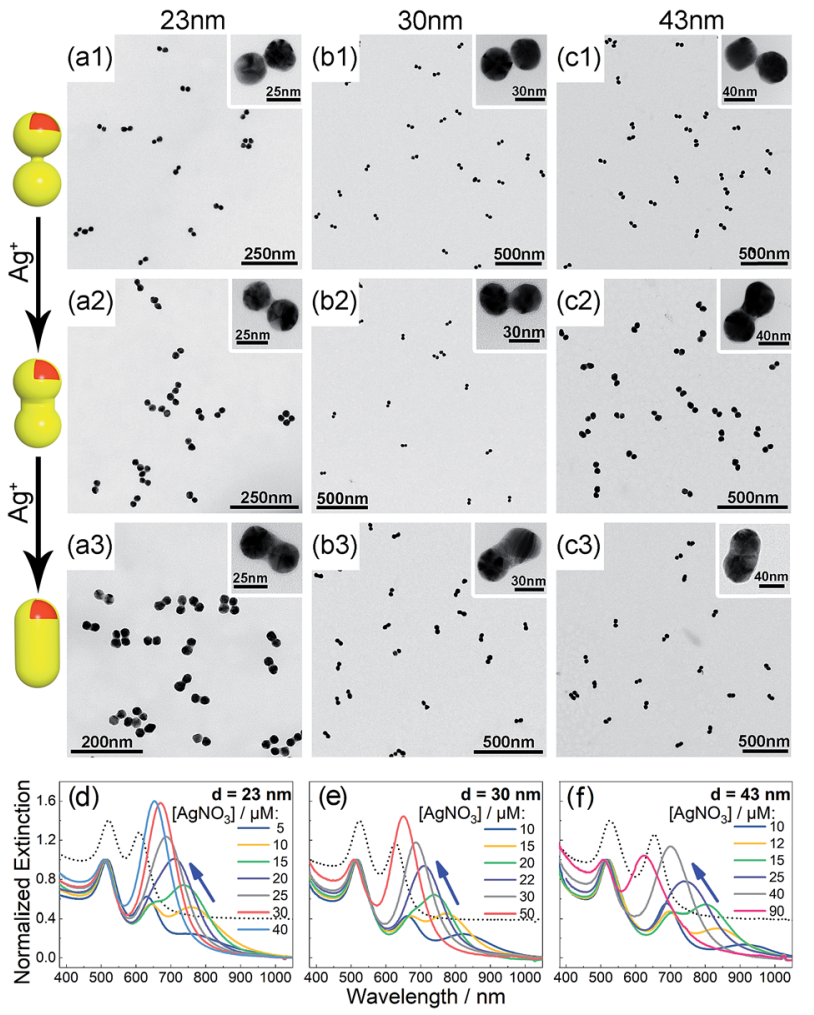

Fig. $2(a-c)$ TEM images of the as-synthesized s-dimers with increased Ag deposition (1-3) based on 23 (a), 30 (b), and $43 \mathrm{~nm}$ (c) AuNPs. Insets are magnified views of the CMNFs. (d-f) Extinction spectra of AuNP dimers after silver deposition to form tunable CTPs. Dotted curves indicate the BDP peaks (located at the right side of the $520 \mathrm{~nm}$ resonances) of unmodified dimers.

obvious for the s-dimers due to the high diffusivity of $\mathrm{Ag}$ atoms. ${ }^{55}$ Such a growth mode was also supported by energy dispersive X-ray (EDX) spectral analysis (ESI, Fig. S12†) of the sdimer product.

A visual sign for the formation of Au and Ag CJs was based on CTP-induced solution color transitions (ESI, Fig. S13 and S14†) due to plasmonic light scattering and absorbance. To rule out aggregation-induced color changes and any color mixing effects caused by structural heterogeneity, agarose gel electrophoresis (AGE) was conducted. All samples appeared as single, sharp gel bands with characteristic colors identical to their native solutions (ESI, Fig. S15 and S16 $)$ ), which exclusively verified that the as-observed colors were from monodisperse CMNFs. Consistently, extinction spectra (Fig. 1 and 2, panels d-f) showed CTP resonances at significantly longer wavelengths than those of the gap-mode BDPs. These CTP peaks gradually shifted from the NIR to visible region when more $\mathrm{Au}$ and $\mathrm{Ag}$ deposits entered the nanofoci. The spectral trend was reasonable as a thickened CJ allows higher frequency electron shuttling between two AuNPs. ${ }^{20,21,24,26,27}$ Meanwhile, the BDP immediately diminished upon the formation of CJs due to charge neutralization. ${ }^{21-27}$ The CTP frequency depends on the junction width $(w)$ and the NP size $(d)$, and thus a small $w / d$ results in a long CTP wavelength. Theoretical plasmonic responses of the g-and s-dimers were obtained by solving full Maxwell's equations with a boundary element method (BEM). ${ }^{56}$ The CJ widths were optimized during the simulations to fit experimental CTPs. A good match was achieved between theoretical and measured CJ widths (ESI, Fig. S17†). We further relied on simulations to reveal different CTP behaviors of the CMNFs with metal layers confined in the gap or on the whole dimer surface (ESI, Fig. S18†). These two scenarios produced similar CTP maxima.

The as-prepared s-dimers were sensitive to oxidative etching by $\mathrm{H}_{2} \mathrm{O}_{2}$ which caused a redshift of their CTPs back to the NIR region (ESI, Fig. S19 and S20†). TEM images evidenced the $\mathrm{H}_{2} \mathrm{O}_{2}$ etched CMNFs (ESI, Fig. S21 †). Interestingly, even in the presence of excess $\mathrm{H}_{2} \mathrm{O}_{2}$, the CTP peak did not completely disappear. This fact indicated high stability of some residual silver due to the electron-drawing effect from the underlying gold, ${ }^{57,58}$ which was responsible for maintaining the conductive contact between two AuNPs. Therefore, we could easily achieve a CTP close to $1000 \mathrm{~nm}$ without breaking the dimeric assembly. Because $\mathrm{H}_{2} \mathrm{O}_{2}$ is a common reactant or product of enzymatic reactions, such a CTP tuning would find applications in chemical and biological sensing.

The gold dimers are ideal antennas capable of concentrating light in their nanogaps. We therefore expected to realize unified plasmonic and catalytic functions in a single CMNF structure. Such structures are valuable for plasmon-enabled in situ Raman probing of catalytic conversions. One key challenge was to introduce catalyst materials into the nanosized gaps without causing a significant loss of their light focusing ability. The sdimers allowed us to realize such otherwise hard-to-make structures by galvanic displacement of pre-formed $\mathrm{Ag}$ junctions with Pt and Pd catalysts. The metal displacement was monitored based on a redshift of the CTP peak due to reduced CJ conductance (ESI, Fig. S22 $\dagger$ ). Finalization of this process was verified by $\mathrm{H}_{2} \mathrm{O}_{2}$-etching which did not cause further spectral changes due to silver loss. To facilitate TEM observations, sdimers with relatively thick Ag CJs were initially employed. Fig. 3 verifies the formation of discrete Pt and Pd deposits in and outside of the nanofoci along with the disappearance of the

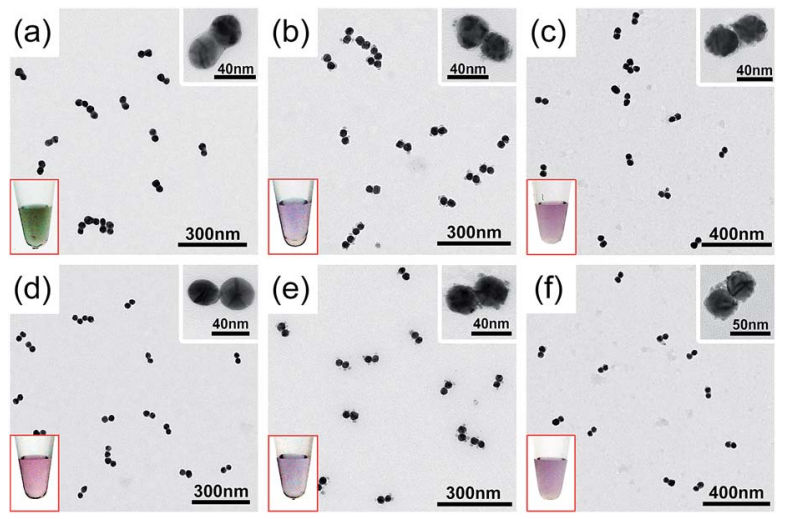

Fig. 3 TEM images showing the s-dimers ( $a$ and d), Pt-s-dimers ( $b$ and e), and Pd-s-dimers ( $c$ and f) before $(a-c)$ and after $(d-f) \mathrm{H}_{2} \mathrm{O}_{2}$ etching to verify the displacement of silver. Insets are magnified TEM views of the CMNFs and photos of their solutions. 
Ag CJs after the galvanic displacements. Despite the successful $\mathrm{Pt} / \mathrm{Pd}$ modifications, the resulting CMNFs had significantly weakened BDP unsuitable for light focusing. We therefore employed s-dimers bearing the thinnest Ag CJs (also improved regioselectivity of $\mathrm{Ag}$ deposition) to minimize gap expansions, which was justifiable since catalytic activities mostly come from several atomic layers of a catalyst phase. While these s-dimers caused difficulty in visualizing the Pt/Pd deposits (ESI, Fig. S23 $\dagger$ ), we could confidently judge the metal displacement from the weakened and redshifted CTP profiles (ESI, Fig. S24 $\dagger$ ). The as-produced CMNFs (termed Pt-s-dimer and Pd-s-dimer) could catalyze the hydrogenation of 4-nitrothiophenol (4-NTP) at the nanofoci, and give plasmonic nanogap-enhanced Raman signals in the meantime.

Three SERS vibrations of 4-NTP at 1090, 1340, and $1573 \mathrm{~cm}^{-1}$ were observed for AuNP dimers and the corresponding CMNF derivatives, corresponding to $\mathrm{C}-\mathrm{S}$ and $\mathrm{O}-\mathrm{N}-\mathrm{O}$ stretching, and phenol-ring breathing modes, respectively (Fig. 4 and $\mathrm{S} 25$ in ESI $\dagger$ ). ${ }^{59}$ At high 4-NTP coverage (incubated with $10 \mu \mathrm{M} 4$-NTP to achieve close-to-saturation adsorption), the AuNP dimers and sdimers only showed slightly attenuated SERS intensities due to $\mathrm{NaBH}_{4}$-induced 4-NTP desorption. In sharp contrast, the Pt-sdimers showed quick diminishing of the 1340 and $1573 \mathrm{~cm}^{-1}$ peaks upon adding $\mathrm{NaBH}_{4}$ at a much higher 4-NTP incubation concentration $(500 \mu \mathrm{M})$ to achieve close-to-saturation adsorption. Such a change was accompanied by the appearance of a new vibration at $1593 \mathrm{~cm}^{-1}$, corresponding to the phenol-ring mode of 4-ATP (4-aminothiophenol, a reduced form of 4-NTP). The final product had a SERS profile similar to that of 4-ATP (ESI, Fig. S26 $\dagger$ ), mirroring a selective hydrogenation of the nitro group. At lowered 4-NTP coverage, the dimers and all CMNFs gave SERS signals reflecting the consumption of 4-NTP along

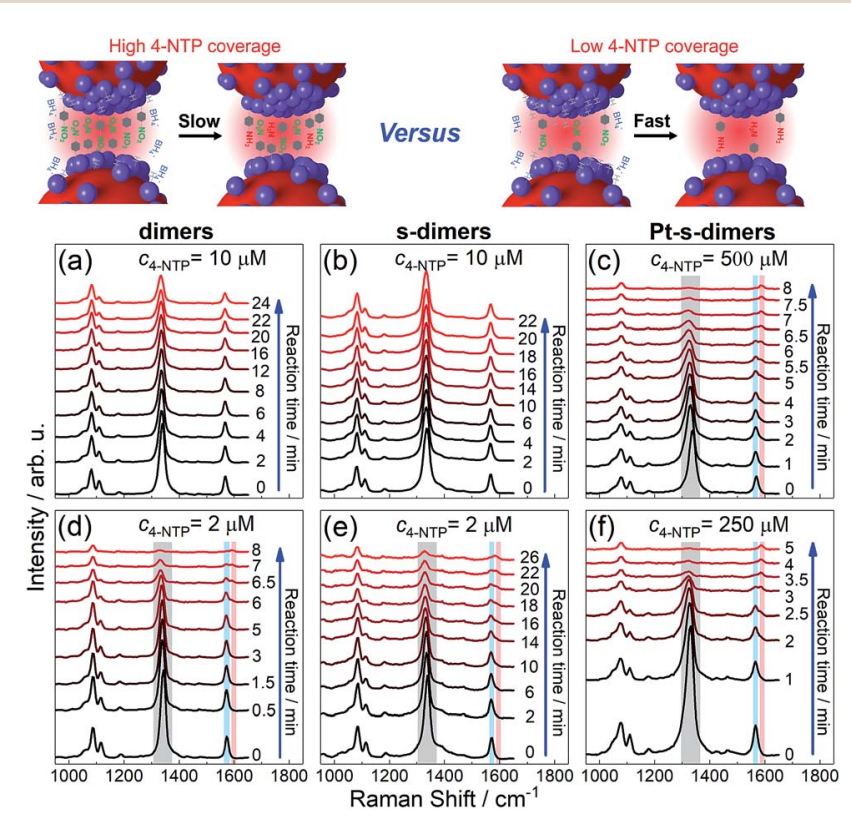

Fig. 4 SERS tracking of $\mathrm{NaBH}_{4}$-based hydrogenation of 4-NTP adsorbed on AuNP dimers ( $a$ and $d$ ), s-dimers ( $b$ and e), and Pt-sdimers ( $c$ and $f$ ) with 4-NTP incubation concentrations indicated on each panel. See Fig. S25† for kinetic SERS data of Pd-s-dimers. with the concomitant formation of 4-ATP. Note that the 4-NTP was only diluted by half as shown in Fig. 4(f), otherwise it would be hard to track the very quick reaction. The weaker reactivity at high 4-NTP coverage was attributed to the self-passivation of catalytic sites by 4-NTP which blocked away the co-adsorptive $\mathrm{BH}_{4}{ }^{-}$and hydrogen species. The inertness of s-dimers contrasted sharply with Pt- and Pd-s-dimers, providing extra evidence for the successful Pt and Pd modifications. Both extinction and TEM data showed negligible alterations to the reacted CMNFs (ESI, Fig. S27 and S28 $\dagger$ ), which guaranteed the reliability of our SERS measurements.

It should be mentioned that the SERS signals were from homogeneously dispersed solution samples with good reproducibility. The 4-ATP was a product of chemical catalysis such that its yield clearly depended on the incubation time between 4-NTP and $\mathrm{NaBH}_{4}$ (ESI, Fig. S29†). The plasmon-driven dimerization of 4-NTP into 4,4'-dimercaptoazobenzene (DMAB) could also be monitored by SERS in a different way with the CMNFs being deposited on a solid substrate. The immobilized CMNFs avoided the diffusion of DMAB out of the focus of the Raman probing laser (ESI, Fig. S30†). Note that the plasmon-induced process did not interfere with the SERS-based monitoring of the chemical catalysis due to different sample preparations (solid vs. solution-based samples).

Considering the unrivaled programmability of DNA in making self-assembled structures, ${ }^{35-37}$ it is highly desired to make our strategy compatible with DNA nanotechnology. To demonstrate this possibility, DNA-linked AuNP dimers were treated by AIS to achieve a strong coupling. ${ }^{46}$ The AIS treatment helped the formation of an Au or Ag CJ between two AuNPs during metal deposition (ESI, Fig. S31 $\dagger$ ). In contrast, DNAlinked dimers without the AIS treatment completely dissociated due to heat-denaturation. Optical extinctions revealed a big difference between the products with and without the AIS treatment (ESI, Fig. S32 $\dagger$ ). Besides, DNA conjugation was possible for the g-dimers to enable DNA-programmable coresatellite assembly (ESI, Fig. S33†).

\section{Conclusions}

In conclusion, we have successfully realized a novel family of chemically modified nanofoci with accurately definable and unified plasmonic and catalytic activities. The conductively linked gold nanodimers by $\mathrm{Au}$ or $\mathrm{Ag}$ junctions lead to charge transfer plasmons with tunable wavelengths up to $1000 \mathrm{~nm}$. By in situ conversion of the silver junctions into Pt and Pd deposits via galvanic displacement, catalytic functions are integrated into the nanofoci without causing a loss of their light focusing ability. The catalytic CMNFs allow real-time, noninvasive, and on-site monitoring of chemical reactions inside the plasmonic nanogaps. The chemical modifications are compatible with DNA-bonded nanoassemblies, leading to DNA-programmable CMNFs. The ability to accurately engineer the plasmonic and catalytic functions of a self-assembled structure opens a new door to sensing, catalysis, spectral enhancements, and photochemistry. The next goal is to pursue light-driven (hotspotenhanced) unconventional chemical pathways benefiting from 
the strongly focused light field unified with prescribed catalytic activities. Compared to nanoparticle aggregates or surfacedeposited colloidal layers, the CMNFs are advantageous due to strong plasmonic coupling, structural purity, and chemical modification specificity. These features make them highly valued for a deep integration between plasmonics and chemistry with minimum ambiguity in structure-function relationships. The structural uniformity may also enable plasmonic and catalytic studies at the single nanoparticle level.

\section{Conflicts of interest}

There are no conflicts of interest to declare.

\section{Acknowledgements}

This work was supported by the National Key Research and Development Program of China (2016YFA0201300), the National Science Fund for Distinguished Young Scholars (21425521), the Foundation for Innovative Research Groups of the NNSFC (21521001), and the Collaborative Innovation Center of Suzhou Nano Science and Technology.

\section{Notes and references}

1 N. Jiang, X. Zhuo and J. Wang, Chem. Rev., 2018, 118, 30543099.

2 E. Hutter and J. H. Fendler, Adv. Mater., 2004, 16, 1685-1706.

3 V. Giannini, A. I. Fernández-Domínguez, S. C. Heck and S. A. Maier, Chem. Rev., 2011, 111, 3888-3912.

4 N. Engheta, Science, 2007, 317, 1698-1702.

5 N. Liu, F. Wen, Y. Zhao, Y. Wang, P. Nordlander, N. J. Halas and A. Alù, Nano Lett., 2013, 13, 142-147.

6 E. M. Roller, L. V. Besteiro, C. Pupp, L. K. Khorashad, A. O. Govorov and T. Liedl, Nat. Phys., 2017, 13, 761-765.

7 S. A. Maier, P. G. Kik, H. A. Atwater, S. Meltzer, E. Harel, B. E. Koel and A. A. G. Requicha, Nat. Mater., 2003, 2, 229232.

8 Y. Nishijima, L. Rosa and S. Juodkazis, Opt. Express, 2012, 20, 11466-11477.

9 N. Zhou, V. López-Puente, Q. Wang, L. Polavarapu, I. Pastoriza-Santos and Q. H. Xu, RSC Adv., 2015, 5, 2907629097.

10 J. R. Mejía-Salazar and O. N. Oliveira Jr, Chem. Rev., 2018, 118, 10617-10625.

11 K. M. Mayer and J. H. Hafner, Chem. Rev., 2011, 111, 38283857.

12 Y. Su, T. Peng, F. Xing, D. Li and C. Fan, Acta Chim. Sin., 2017, 75, 1036-1046.

13 E. Boisselier and D. Astruc, Chem. Soc. Rev., 2009, 38, 17591782.

14 G. Chen, I. Roy, C. Yang and P. N. Prasad, Chem. Rev., 2016, 116, 2826-2885.

15 S. Linic, U. Aslam, C. Boerigter and M. Morabito, Nat. Mater., 2015, 14, 567-576.

16 J. Y. Park, S. M. Kim, H. Lee and I. I. Nedrygailov, Acc. Chem. Res., 2015, 48, 2475-2483.
17 I. I. Nedrygailov, H. Lee, S. W. Lee and J. Y. Park, Chin. Chem. Lett., 2018, 29, 727-733.

18 E. Prodan, C. Radloff, N. J. Halas and P. Nordlander, Science, 2003, 302, 419-422.

19 P. Nordlander and C. Oubre, Nano Lett., 2004, 4, 899-903.

20 N. J. Halas, S. Lal, W. S. Chang, S. Link and P. Nordlander, Chem. Rev., 2011, 111, 3913-3961.

21 I. Romero, J. Aizpurua, G. W. Bryant and F. J. G. de Abajo, Opt. Express, 2006, 14, 9988-9999.

22 N. Liu, M. L. Tang, M. Hentschel, H. Giessen and A. P. Alivisatos, Nat. Mater., 2011, 10, 631-636.

$23 \mathrm{~W}$. Zhu, R. Esteban, A. G. Borisov, J. J. Baumberg, P. Nordlander, H. J. Lezec, J. Aizpurua and K. B. Crozier, Nat. Commun., 2016, 7, 11495.

24 K. J. Savage, M. M. Hawkeye, R. Esteban, A. G. Borisov, J. Aizpurua and J. J. Baumberg, Nature, 2012, 491, 574-577.

25 R. Esteban, A. G. Borisov, P. Nordlander and J. Aizpurua, Nat. Commun., 2012, 3, 825.

26 O. Pérez-González, N. Zabala, A. G. Borisov, N. J. Halas, P. Nordlander and J. Aizpurua, Nano Lett., 2010, 10, 30903095.

27 T. Atay, J. H. Song and A. V. Nurmikko, Nano Lett., 2004, 4, 1627-1631.

28 S. F. Tan, L. Wu, J. K. W. Yang, P. Bai, M. Bosman and C. A. Nijhuis, Science, 2014, 343, 1496-1499.

29 Y. Sun, J. J. Foley IV, S. Peng, Z. Li and S. K. Gray, Nano Lett., 2013, 13, 3958-3964.

30 J. H. Lee, M. H. You, G. H. Kim and J. M. Nam, Nano Lett., 2014, 14, 6217-6225.

31 W. Xu, Z. Li and Y. Yin, Small, 2018, 14, 1801083.

32 Y. Hu and Y. Sun, J. Am. Chem. Soc., 2013, 135, 2213-2221.

33 M. Lin, G. H. Kim, J. H. Kim, J. W. Oh and J. M. Nam, J. Am. Chem. Soc., 2017, 139, 10180-10183.

34 Z. Wang, B. He, G. Xu, G. Wang, J. Wang, Y. Feng, D. Su, B. Chen, H. Li, Z. Wu, H. Zhang, L. Shao and H. Chen, Nat. Commun., 2018, 9, 563.

35 N. C. Seeman, Nature, 2003, 421, 427-431.

36 M. R. Jones, N. C. Seeman and C. A. Mirkin, Science, 2015, 347, 1260901.

37 N. Liu and T. Liedl, Chem. Rev., 2018, 118, 3032-3053.

38 J. Y. Kim and N. A. Kotov, Chem. Mater., 2014, 26, 134-152. 39 L. Song and Z. Deng, ChemNanoMat, 2017, 3, 698-712.

40 A. Thill and O. Spalla, Langmuir, 2002, 18, 4783-4789.

41 P. A. Kralchevsky and K. Nagayama, Langmuir, 1994, 10, 2336.

42 Z. Zhou, Q. Li and X. S. Zhao, Langmuir, 2006, 22, 3692-3697. 43 Y. B. Vogel, V. R. Gonçales, L. Al-Obaidi, J. J. Gooding, N. Darwish and S. Ciampi, Adv. Funct. Mater., 2018, 28, 1804791.

44 Y. B. Vogel, J. Zhang, N. Darwish and S. Ciampi, ACS Nano, 2018, 12, 8071-8080.

45 M. Liu, L. Fang, Y. Li, M. Gong, A. Xu and Z. Deng, Chem. Sci., 2016, 7, 5435-5440.

46 H. Wang, Y. Li, M. Liu, M. Gong and Z. Deng, Small, 2015, 11, 2247-2251.

47 L. Fang, Y. Wang, M. Liu, M. Gong, A. Xu and Z. Deng, Angew. Chem., Int. Ed., 2016, 128, 14508-14512. 
48 L. Fang, D. Liu, Y. Wang, Y. Li, L. Song, M. Gong, Y. Li and Z. Deng, Nano Lett., 2018, 18, 7014-7020.

49 S. Ding, J. Yi, J. Li, B. Ren, D. Wu, R. Panneerselvam and Z. Tian, Nat. Rev. Mater., 2016, 1, 16021.

50 C. Wang, L. Tian, W. Zhu, S. Wang, N. Gao, K. Zhou, X. Yin, W. Zhang, L. Zhao and G. Li, Chem. Sci., 2018, 9, 889-895.

51 U. Aslam, S. Chavez and S. Linic, Nat. Nanotechnol., 2017, 12, 1000-1005.

52 A. S. Alnaser, M. Kübel, R. Siemering, B. Bergues, N. G. Kling, K. J. Betsch, Y. Deng, J. Schmidt, Z. A. Alahmed, A. M. Azzeer, J. Ullrich, I. Ben-Itzhak, R. Moshammer, U. Kleineberg, F. Krausz, R. de Vivie-Riedle and M. F. Kling, Nat. Commun., 2014, 5, 3800.
53 A. C. Aragonès, N. L. Haworth, N. Darwish, S. Ciampi, N. J. Bloomfield, G. G. Wallace, I. Diez-Perez and M. L. Coote, Nature, 2016, 531, 88-91.

54 T. Cosgrove, Colloid Science: Principles, Methods and Applications, John Wiley \& Sons, West Sussex, 2010.

55 W. C. Mallard, A. B. Gardner, R. F. Bass and L. M. Slifkin, Phys. Rev., 1963, 129, 617-625.

56 U. Hohenester and A. Trügler, Comput. Phys. Commun., 2012, 183, 370-381.

57 H. Wang, Y. Li, M. Gong and Z. Deng, Chem. Sci., 2014, 5, 1015-1020.

58 D. T. N. Anh, P. Singh, C. Shankar, D. Mott and S. Maenosono, Appl. Phys. Lett., 2011, 99, 073107.

59 W. Xie and S. Schlücker, Chem. Commun., 2018, 54, 23262336. 